\title{
In vivo Raman analysis of human skin lesions
}

\author{
Ali Tfayli, Olivier Piot, Sylvie Derancourt, Philippe Bernard, \\ and Michel Manfait
}

A new Raman system, consisting of optic fiber bundles coupled to an axial Raman spectrometer has been developed for in-vivo investigations and can be used on human skin lesions.

Vibrational spectroscopy is increasingly used for the investigation of biological tissues: spectral analysis reveals hidden information at microstructural and molecular levels. Moreover, in vitro and ex vivo applications have established the potential of these techniques for detecting and diagnosing different types of lesions. Several studies show that it can successfully differentiate cancerous from benign tissues on thin sections of biopsies on colon, cervix, stomach, breast, and skin. ${ }^{1-11}$ For in vivo diagnosis, Raman spectroscopy permits a non-destructive analysis, unhindered by the aqueous nature of tissues. It does not require any previous sample preparation or labeling. In addition, it is a rapid technique enabling real-time spectral acquisition.

In 1997 M.G. Shim et al. ${ }^{12}$ described a fiber-based Raman system and presented in vivo spectra from buccal cheek epithelium, human skin, fingernail, and tooth. Since then, several other invivo studies have been done on atherosclerotic plaque, ${ }^{13}$ skin, ${ }^{14}$ colon ${ }^{15}$ and esophagus. ${ }^{15}$ For our work we used a new spectrometer, the unique feature of which is its size $(60 \mathrm{~cm}$ long). This allows for easy transport into operation rooms for in-situ clinical application. We used the devices to perform a study on 10 consenting patients treated for basal cell carcinomas (BCC), a very common skin cancer.

The spectrometer has an axial geometry with no moving parts. It is equipped with a laser diode emitting at $830 \mathrm{~nm}$, a bifurcated fiber-optic bundle (SEDI Fibers) to transport excitation and scattered light, a grism (grating and prism) to spread the light, and a charge-coupled device (CCD) detector (see Figure 1).

The size of the fiber bundle was limited to a $1.6 \mathrm{~mm}$ diameter so it could be inserted into an endoscope. The spectral resolution is $2 \mathrm{~cm}^{-1}$ and the output power at the central delivery fiber is $110 \mathrm{~mW}$. These specifications mean the system is safe for in-vivo application. However, it can acquire a Raman spectrum in just 30 seconds.

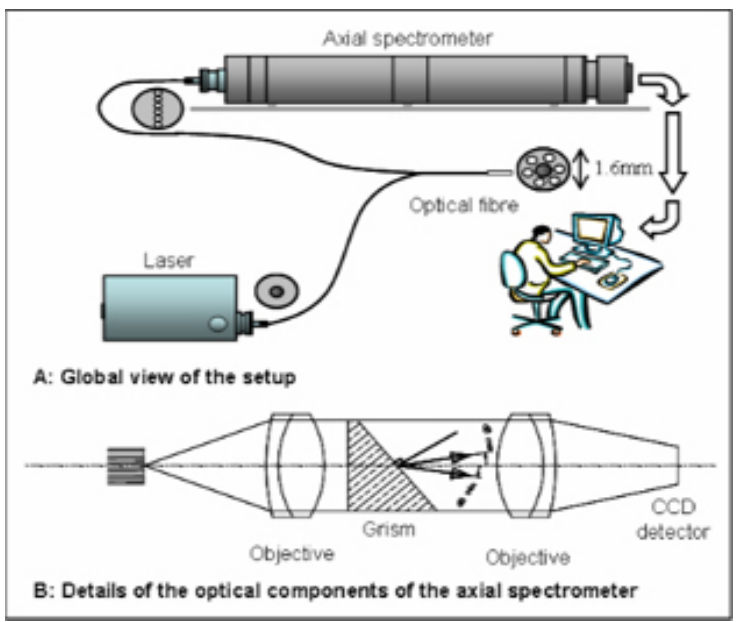

Figure 1. A Global schematic view of the setup (a), and detailed view of the axial spectrometer $(b)$.

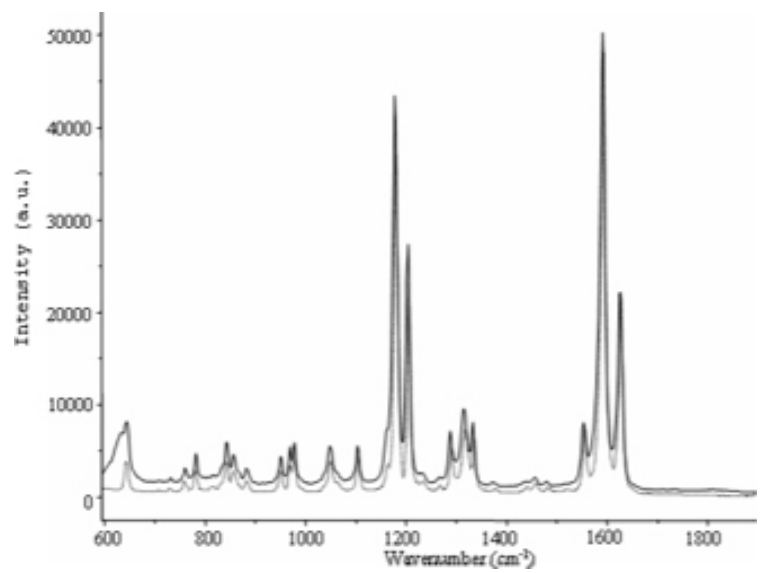

Figure 2. The spectra of benzene recorded with the Labram (dotted line) and the portable axial spectrometer (connected line).

The first step in the measurement process required the verification of the system efficiency, accuracy, and resolution. To this end, we collected spectra of pure products like benzene

Continued on next page 
and naphthalene commonly used as reference products in Raman spectroscopy (see Figure 2). Then, we collected spectra from phenylalanine and tyrosine (see Figure 3), two amino acids that present highly intense bands that can easily be identified all tissue spectra. Finally we collected the signal from elastin fibers (not shown). In both Figure 2 and 3 the quality of spectra is weakly affected by the fiber signal. For elastin, the signal-tonoise ratio decreased significantly as compared to a Labram spectrometer spectra, also from the Horiba Jobin Yvon company.

In-vivo skin spectra present an intense signal below $1100 \mathrm{~cm}^{-1}$ due to the silica in the fiber, and this needed to be corrected before any classification. The spectral windows were therefore reduced to the $1000-1800 \mathrm{~cm}^{-1}$ region, where the silica fiber contribution can be mathematically eliminated via a polynomial base-

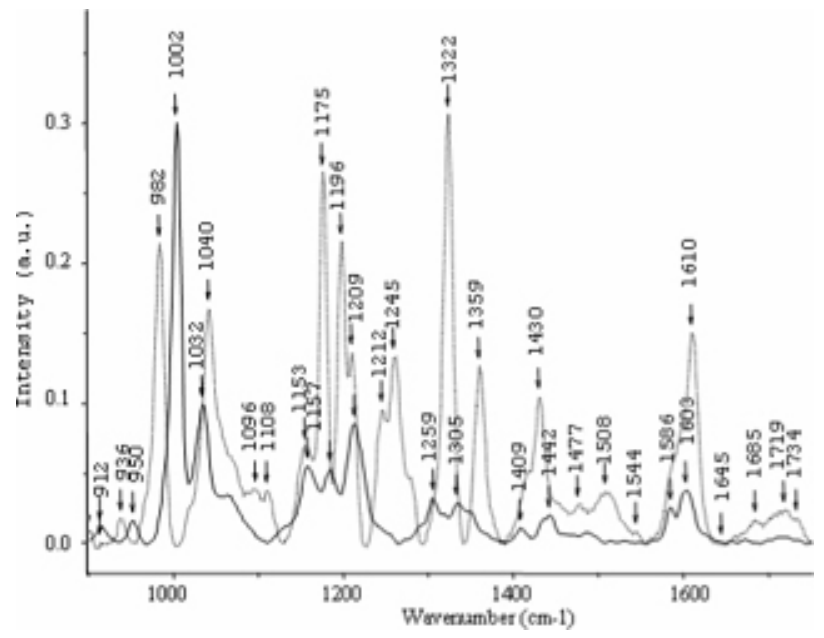

Figure 3. The spectra of phenylalanine (connected line) and tyrosine (dotted line) in the $900-1750 \mathrm{~cm}^{-1}$ region recorded with the portable axial spectrometer.

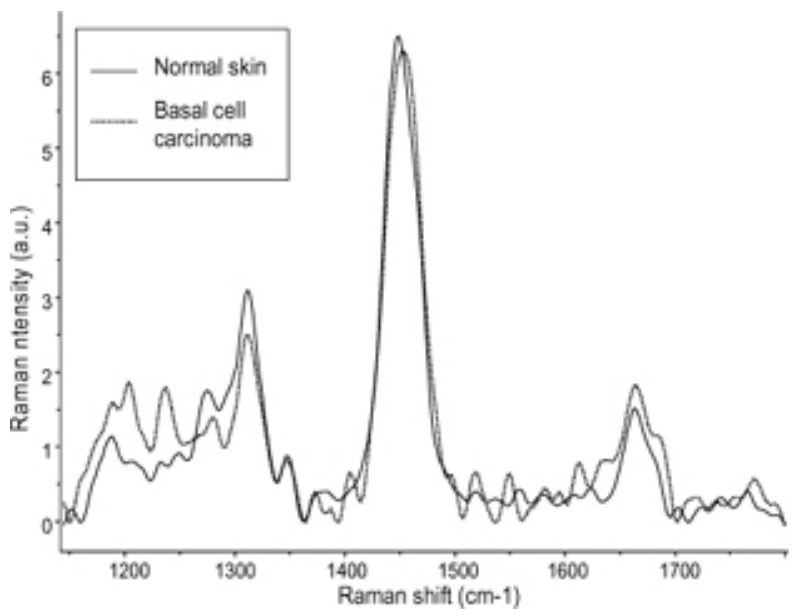

Figure 4. A comparison between the mean spectrum of normal skin and the basal cell carcinoma mean spectrum.

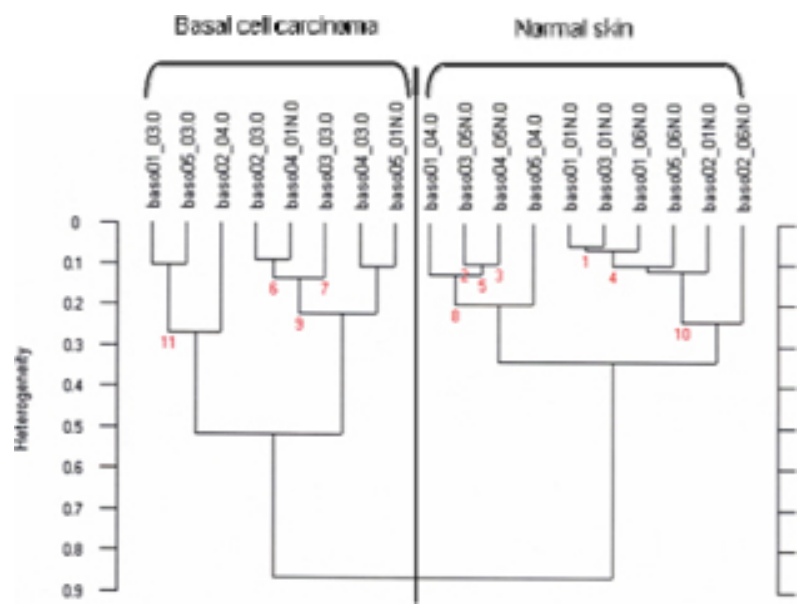

Figure 5. A dendrogram separating into two groups the spectra of normal and cancerous skin.

line correction. All skin spectra were corrected using the same function.

Hierarchical cluster analysis (HCA) was performed to classify the spectral data. A thorough description of this statistical analysis can be found in Reference 11. The results are shown in a tree-like diagram (dendrogram), grouping spectra in clusters according to a heterogeneity scale (see Figure 5)

Comparison between normal skin and BCC spectra (see Figure 4) shows a slight frequency shift in the $\mathrm{C}-\mathrm{H}$ deformation mode (around $1450 \mathrm{~cm}^{-1}$ ), a weak shoulder at $1685 \mathrm{~cm}^{-1}$ in the protein Amide I band of carcinoma spectra, and some differences in the $1200-1250 \mathrm{~cm}^{-1}$ range (Amide III and phospholipids). HCA was performed on the Amide-III band and the C-H deformation band at $1410 \mathrm{~cm}^{-1}$.

In spite of the poor quality of skin signal (because of the high contribution of the fused silica fiber), the combination of spectral data and HCA enables a relatively good separation.

\section{Conclusion and perspectives}

The examples presented here show that our portable Raman system could potentially be used as diagnostic tool in medical care centers. Improvements in optical instrumentation are possible by putting the appropriate filters at the tip of the bundle and by changing fiber geometry. Also, more advanced data analysis should permit improved signal quality by creating models of the fiber signal and therefore allowing for more accurate cancellation of its effect.

Further experiments will involve a larger number of patients with respect to variations in skin lesions. Sex, age, and location on the body will also be taken into account. 


\section{Author Information}

\section{Ali Tfayli, Olivier Piot, and Michel Manfait}

MéDIAN unit

UFR Pharmacy, University of Reims

Reims, France

\section{Sylvie Derancourt and Philippe Bernard}

Dermatology Service

CHU Robert Debré

Reims, France

\section{References}

1. N. Stone, C. Kendall, J. Smith, P. Crow, and H. Barr, Raman spectroscopy for identification of epithelial cancers, Faraday Discuss. 126, pp. 169-183, 2004.

2. P. Lasch, W. Haensch, D. Naumann, and M. Diem, Imaging of colorectal adenocarcinoma using FT-IR microspectroscopy and cluster analysis, Biochim. Biophys. Acta 1688, pp. 176-186, 2004.

3. Q. B. Li, Z. Xu, N. W. Zhang, L. Zhang, F. Wang, L. M. Yang, J. S. Wang, S. Zhou, Y. F. Zhang, X. S. Zhou, J. S. Shi, and J. G. Wu, In vivo and in situ detection of colorectal cancer using Fourier transform infrared spectroscopy, World J. Gastroenterol 11, pp. 327-330, 2005.

4. B. R. Wood, L. Chiriboga, H. Yee, M. A. Quinn, D. McNaughton, and M. Diem, Fourier transform infrared (FTIR) spectral mapping of the cervical transformation zone, and dysplastic squamous epithelium, Gynecol. Oncol. 93, pp. 59-68, 2004.

5. S. Mordechai, R. K. Sahu, Z. Hammody, S. Mark, K. Kantarovich, H. Guterman, A. Podshyvalov, J. Goldstein, and S. Argov, Possible common biomarkers from FTIR microspectroscopy of cervical cancer and melanoma, J. Microsc. 215, pp. 86-91, 2004.

6. X. Ling, Y. Xu, S. Weng, W. Li, Z. Xu, R. Hammaker, W. Fateley, F. Wang, X. Zhou, R. Soloway, J. Ferraro, and J. Wu, Investigation of normal and malignant tissue samples from the human stomach using Fourier Transform Raman spectroscopy, Applied Spectroscopy 53, 2002.

7. N. Fujioka, Y. Morimoto, T. Arai, and M. Kikuchi, Discrimination between normal and malignant human gastric tissues by Fourier transform infrared spectroscopy, Cancer Detect. Prev. 28, pp. 32-36, 2004

8. H. Fabian, P. Lasch, M. Boese, and W. Haensch, Mid-IR microspectroscopic imaging of breast tumor tissue sections, Biopolymers 67, pp. 354-357, 2002.

9. R. Mendelsohn, H. C. Chen, M. E. Rerek, and D. J. Moore, Infrared microspectroscopic imaging maps the spatial distribution of exogneous molecules in the skin, J. Biomed. Opt. 8, pp. 185-90, 2003.

10. M. Gniadecka, P. A. Philipsen, S. Sigurdsson, S. Wessel, O. F. Nielsen, D. H. Christensen, J. Hercogova, K. Rossen, H. K. Thomsen, R. Gniadecki, L. K. Hansen, and H. C. Wulf, Melanoma diagnosis by Raman spectroscopy and neural networks: structure alterations in proteins and lipids in intact cancer tissue, J. Invest. Dermatol. 122, pp. 443-449, 2004.

11. A. Tfayli, O. Piot, A. Durlach, P. Bernard, and M. Manfait, Discriminating nevus and melanoma on paraffin-embedded skin biopsies using FTIR microspectroscopy, Biochim. Biophys. Acta 1724, pp. 262-269, 2005.

12. M. G. Shim and B. C. Wilson, Development of an in vivo Raman spectroscopic system for diagnostic applications, J. Raman Spectroscopy 28, pp. 131-142, 1997.

13. J. T. Motz, M. Hunter, L. H. Galindo, J. A. Gardecki, J. R. Kramer, R. R. Dasari, and M. S. Feld, Optical fiber probe for biomedical spectroscopy, Applied Optics 43 (3), 2004.

14. L. Chrit, C. Hadjur, S. Morel, G. Sockalinghum, G. Lebourdon, F. Leroy, and M. Manfait, In vivo chemical investigation of human using a confocal Raman fiber optic microprobe, J. Biomed. Opt. 10 (4), 2005

15. M. G. Shim, L. M. Wong Kee Song, N. E. Marcon, and B. Wilson, In vivo nearinfrared Raman spectroscopy: demonstration of feasibility during clinical gastrointestinal endoscopy, Photochem. Photobiol. 72 (1), pp. 146-150, 2000. 